\title{
Adaptive Control And Single Parameter Identification Of One Order System
}

\author{
Yuqiang $\mathrm{Jin}^{1, \mathrm{a}}$, Zexue $\mathrm{Li}^{2}$ and Junwei Lei ${ }^{2, \mathrm{~b}}$ \\ ${ }^{1}$ Department of Training, Naval aeronautical and astronautical University, Yantai, China \\ ${ }^{2}$ Department of control engineering, Naval aeronautical and astronautical University, Yantai, China \\ ajinyuqiang1024@126.com, 'reijunwei@126.com
}

Keywords: adaptive control, parameter identification, one order system, stability, one order system

\begin{abstract}
In order to achieve adaptive control and single parameter identification of one order system, an ordinary adaptive control method is used in this paper. Based on Terminal attractor and Sigmoid function, an adaptive control law is designed, and the result of parameter identification is analyzed. In the end, a conclusion can be made that theoretical analysis is correct and parameter identification method is effective by numerical simulation.
\end{abstract}

\section{Introduction}

As the demand for control performance increases, the problem of time-variance parameters of controlled system is more and more important ${ }^{[1-4]}$. A lot of parameter identification method is proposed by specialists and representatives from China and abroad ${ }^{[5-7]}$. The single parameter identification is a kind of simple situation but it is still has some result that can be found when we research on it ${ }^{[8-11]}$. The classical parameter identification method is least square method ${ }^{[1]}$, Kalman filtering method, adaptive. The method modern parameter identification method is neural network method $^{[2]}$, genetic algorithm method ${ }^{[3]}$ and particle swarm optimization. But calculating amount of these method is large, instantaneity and astringency can't meet the demands of the control system. Based on Terminal attractor and Sigmoid function, an adaptive identification controller is proposed in this paper.

\section{Problem Description}

One order system can be written as:

$$
\dot{x}=a x+u
$$

where $a$ is unknown constant parameter, the goal is designing a controller such that the system state $x$ can trace the expected value $x^{d}$.

\section{Design Adaptive Identification Controller} then

An ordinary adaptive control method is used as follows, define a error variable as $z_{1}=x_{1}-x_{1}^{d}$,

$$
\dot{z}_{1}=\dot{x}_{1}-\dot{x}_{1}^{d}=a x+u
$$

Design state feedback control law as:

$$
u=-\hat{a} x-\sum_{i=1}^{n} k_{i} f_{i}\left(z_{1}\right)
$$

where $n=5$,

$$
\begin{gathered}
f_{1}\left(z_{1}\right)=z_{1}, \quad f_{2}\left(z_{1}\right)=z_{1}^{3}, \quad f_{3}\left(z_{1}\right)=z_{1}^{1 / 3} \\
f_{4}\left(z_{1}\right)=\frac{z_{1}}{\left|z_{1}\right|+\varepsilon}, \quad \varepsilon=0.2,
\end{gathered}
$$




$$
f_{5}\left(z_{1}\right)=\frac{1-e^{-\tau z_{1}}}{1+e^{-\tau z_{1}}}, \quad \tau=0.5
$$

where $f_{3}\left(z_{1}\right)$ is Terminal attractor, and $f_{5}\left(z_{1}\right)$ is Sigmoid function, $f_{4}\left(z_{1}\right)$ and $f_{5}\left(z_{1}\right)$ both have boundedness, Obviously, $f_{i}\left(z_{1}\right)$ meet $z_{1} f_{i}\left(z_{1}\right) \geq 0$, then

$$
\dot{z}_{1}=\tilde{a} x-\sum_{i=1}^{n} k_{i} f_{i}\left(z_{1}\right)
$$

where the error variable $\tilde{a}$ can be defined as:

design regulating law:

$$
\tilde{a}=a-\hat{a},
$$

$$
\dot{\hat{a}}=\Gamma z_{1} x
$$

where $\hat{a}$ is unknown estimated parameter value, choose initial value $\hat{a}(0)=0$, then

$$
\dot{\tilde{a}}=-\dot{\hat{a}}
$$

choose Lyapunov function:

$$
V=\frac{1}{2} z_{1}^{2}+\frac{1}{2 \Gamma} \tilde{a}^{2}
$$

then

$$
\dot{V}=z_{1} \dot{z}_{1}+\frac{1}{\Gamma} \tilde{a} \dot{\tilde{a}}
$$

then

$$
\dot{V}=z_{1} \tilde{a} x-\sum_{i=1}^{n} k_{i} z_{1} f_{i}\left(z_{1}\right)-\frac{1}{\Gamma} \tilde{a} \Gamma z_{1} x=-\sum_{i=1}^{n} k_{i} z_{1} f_{i}\left(z_{1}\right) \leq 0
$$

So $Z_{1} \rightarrow 0$.

\section{Parameter Identification Result Analysis}

When $z_{1} \rightarrow 0$, where $u=-\hat{a} x$, then

$$
\dot{z}_{1}=a x-\hat{a} x=\tilde{a} x
$$

So when $z_{1} \rightarrow 0$, where $\dot{z}_{1} \rightarrow 0, \quad \dot{z}_{1}=a x-\hat{a} x=\tilde{a} x=0$, then $\quad \dot{z}_{1}=a x-\hat{a} x=\tilde{a} x=0$, when $x \neq 0$, where $\tilde{a} \rightarrow 0$.

\section{Numerical Simulation}

Choose $a=5, x_{1}^{d}=2$, program can be written as follows:

clc;clear;a $=5 ; \mathrm{x} 1 \mathrm{~d}=2 ; \mathrm{ag}=0 ; \mathrm{x}=0 ; \mathrm{u}=0 ; \mathrm{tf}=15 ; \mathrm{dt}=0.001$;

for $\mathrm{i}=1: \mathrm{tf} / \mathrm{dt}$

$\mathrm{t}=\mathrm{i} * \mathrm{dt} ; \quad \mathrm{dx}=\mathrm{a} * \mathrm{x}+\mathrm{u} ; \quad \mathrm{x}=\mathrm{x}+\mathrm{dx} * \mathrm{dt}$

$\mathrm{k} 1=5 ; \mathrm{k} 2=0.2 ; \mathrm{k} 3=1 ; \mathrm{k} 4=1 ; \mathrm{k} 5=1 ;$ esten $=0.2 ; \mathrm{tao}=0.5$;

$\mathrm{z} 1=\mathrm{x}-\mathrm{x} 1 \mathrm{~d} ; \quad \mathrm{f} 1=\mathrm{z} 1 ; \mathrm{f} 2=\mathrm{z} 1 \wedge 3 ; \mathrm{f} 3=\mathrm{z} 1 \wedge(1 / 3) ; \mathrm{f} 4=\mathrm{z} 1 /(\operatorname{abs}(\mathrm{z} 1)+e s t e n) ;$

$\mathrm{f} 5=\left(1-\exp \left(-\right.\right.$ tao $\left.\left.^{*} \mathrm{z} 1\right)\right) /\left(1+\exp \left(-\mathrm{tao}^{*} \mathrm{z} 1\right)\right)$;

$\mathrm{ta}=30 ; \quad$ dag $=\mathrm{ta}^{*} \mathrm{x} * \mathrm{z} 1 ; \quad \mathrm{ag}=\mathrm{ag}+\mathrm{dag} * \mathrm{dt}$;

$\mathrm{u}=-\mathrm{ag} * \mathrm{x}-\mathrm{k} 1 * \mathrm{f} 1-\mathrm{k} 2 * \mathrm{f} 2-\mathrm{k} 3 * \mathrm{f} 3-\mathrm{k} 4 * \mathrm{f} 4-\mathrm{k} 5 * \mathrm{f} 5$;

end

tp(i) $=t ; x p(i)=x ; \operatorname{agp}(i)=a g$;

figure(1);plot(tp,xp);xlabel('t/s');ylabel('state x');

figure(2);plot(tp,agp);xlabel('t/s');ylabel('state ag');

And the simulation results are as follows: 


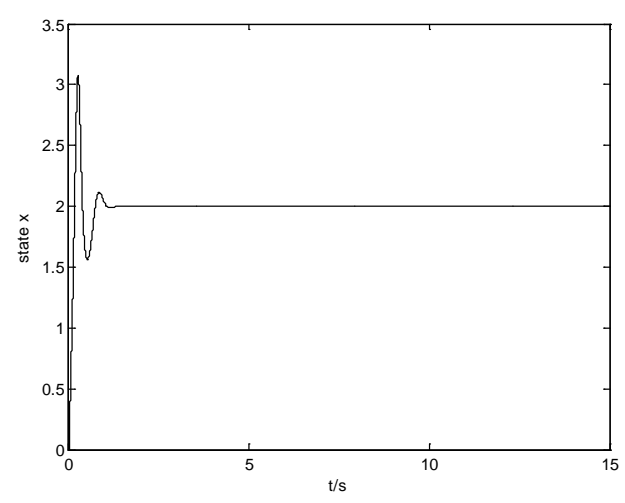

Fig.1 state $\mathrm{x}$

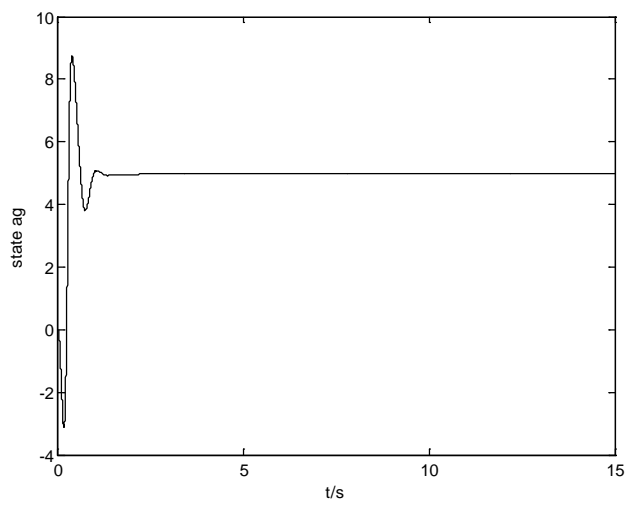

Fig.2 state a

From the simulation results, we can know that the unknown estimated parameter value is close to the actual value, and system state is also close to the expected value, these show that the method in this paper is effective and parameter identification result analysis is correct. But when control gain is not matched with identification gain, parameter identification will cost much time, so the program can be written as:

clc;clear; $\mathrm{a}=5 ; \mathrm{x} 1 \mathrm{~d}=2 ; \mathrm{ag}=0 ; \mathrm{x}=0 ; \mathrm{u}=0 ; \mathrm{tf}=150 ; \mathrm{dt}=0.001$;

for $\mathrm{i}=1: \mathrm{tf} / \mathrm{dt}$

$$
\begin{aligned}
& \mathrm{t}=\mathrm{i} * \mathrm{dt} ; \quad \mathrm{dx}=\mathrm{a} * \mathrm{x}+\mathrm{u} ; \quad \mathrm{x}=\mathrm{x}+\mathrm{dx} * \mathrm{dt} ; \\
& \mathrm{k} 1=5 ; \mathrm{k} 2=0.2 ; \mathrm{k} 3=1 ; \mathrm{k} 4=1 ; \mathrm{k} 5=1 ; \text { esten }=0.2 ; \mathrm{tao}=0.5 \text {; } \\
& \mathrm{k} 1=5 ; \mathrm{k} 2=5 ; \mathrm{k} 3=5 ; \mathrm{k} 4=5 ; \mathrm{k} 5=5 \text {; } \\
& \mathrm{z} 1=\mathrm{x}-\mathrm{x} 1 \mathrm{~d} ; \quad \mathrm{f} 1=\mathrm{z} 1 ; \mathrm{f} 2=\mathrm{z} 1 \wedge 3 ; \mathrm{f} 3=\mathrm{z} 1 \wedge(1 / 3) ; \mathrm{f} 4=\mathrm{z} 1 /(\mathrm{abs}(\mathrm{z} 1)+\text { esten }) \text {; } \\
& \mathrm{f} 5=\left(1-\exp \left(-\mathrm{tao}^{*} \mathrm{z} 1\right)\right) /\left(1+\exp \left(-\mathrm{tao}^{*} \mathrm{z} 1\right)\right) \text {; } \\
& \text { ta }=0.1 ; \quad \text { dag }=\text { ta }^{*} \mathrm{x}^{*} \mathrm{z} 1 ; \quad \mathrm{ag}=\mathrm{ag}+\text { dag*dt; } \\
& \mathrm{u}=-\mathrm{ag} * \mathrm{x}-\mathrm{k} 1 * \mathrm{f} 1-\mathrm{k} 2 * \mathrm{f} 2-\mathrm{k} 3 * \mathrm{f} 3-\mathrm{k} 4 * \mathrm{f} 4-\mathrm{k} 5 * \mathrm{f} 5 \text {; } \\
& \operatorname{tp}(\mathrm{i})=\mathrm{t} ; \mathrm{xp}(\mathrm{i})=\mathrm{x} ; \operatorname{agp}(\mathrm{i})=\mathrm{ag} \text {; }
\end{aligned}
$$

end

figure(1);plot(tp,xp,'k');xlabel('t/s');ylabel('state x');

figure(2);plot(tp,agp,'k');xlabel('t/s');ylabel('state ag');

And the simulation results are as follows:

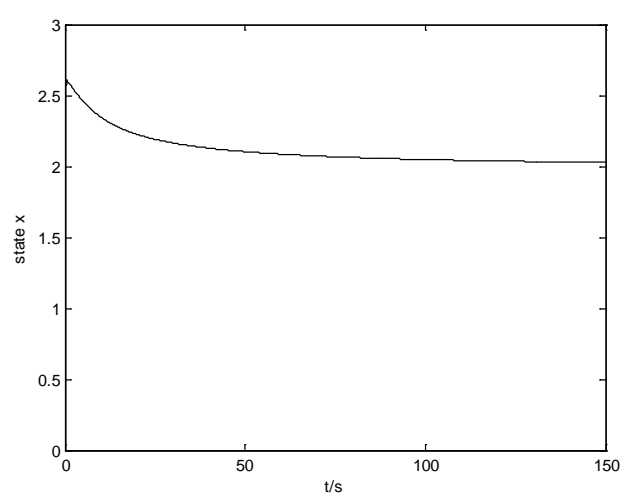

Fig.3 state $\mathrm{x}$

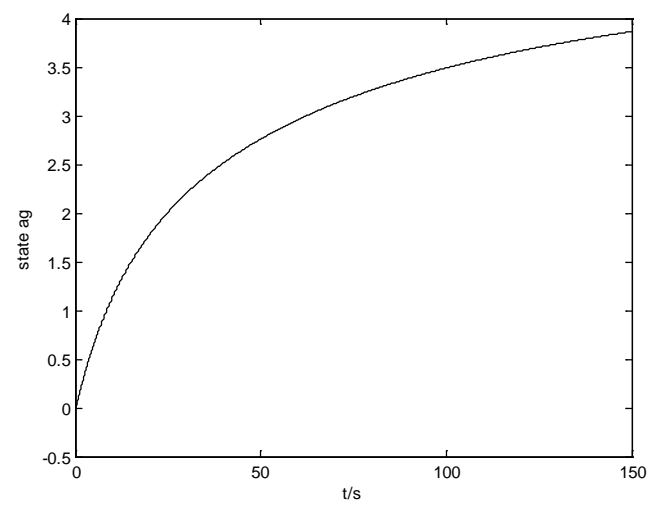

Fig.4 state ag

\section{Conclusion}

By analysing theory and the simulation results, we can make a conclusion that the method of combining Terminal and Sigmoid function in this paper is effective to parameter identification. When the control gain is matched with identification gain, unknown parameter identification can success easily. But when the control gain is not matched with identification gain, the result is not ideal. 


\section{Reference}

[1] Maurizio C, Marcello P, Giansalvo, et al. A new experimental application of least-squares techniques for the estimation of the induction motor parameters[J]. IEEE Transactions on Industry Applications, 2003, 39(5):1247-1256.

[2] Raj M B, Alexander G P, Hamid A T, Neural speed filtering for sensorless induction motor drives[J]. Control Engineering Practice, 2004, 12(6): 687-706.

[3] Huang K S, Kent W, Wu Q, et al. Parameter identification of an induction machine using genetic algorithms[C] // Proceedings of the 1999 IEEE International Symposium on Computer Aided Control System Design. [S. L.]: [s. N.],1999: 510-515.

[4] Huynh D C, Dunnugan M W. Parameter estimation of an induction machine using advanced particle swarm optimisation algorithms[J]. IET Electric Power Applications, 2010, 4(9): 748-760.

[6] Fuh CC, Tung PC. Controlling chaos using differential geometric method. Phys Rev Lett 1995;75:2952-5.

[7] Chen G, Dong X. On feedback control of chaotic continuous-time systems. IEEE Trans Circ Syst 1993;40:591-601.

[8] Sanchez EN, Perez JP, Martinez M, Chen G. Chaos stabilization: an inverse optimal control approach. Latin Amer Appl Res: Int J 2002;32:111-4.

[9] Feki M. An adaptive feedback control of linearizable chaotic systems. Chaos, Solitons \& Fractals 2003;15:883-90.

[10] Wang Y, Guan Z, Wen X. Adaptive synchronization for Chen chaotic system with fully unknown parameters. Chaos, Solitons \&Fractals 2004;19:899-903.

[11] Chen G, Dong X. From chaos to order: methodologies, perspectives and applications. Singapore: World Scientific;1998. 